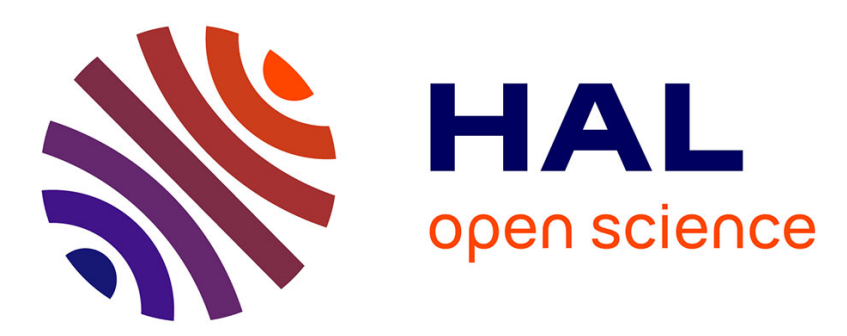

\title{
Utilisation de signaux Doppler tolérants en trajectographie active; essais en mer
}

\author{
M. Zakharia, F. Joly
}

\section{To cite this version:}

M. Zakharia, F. Joly. Utilisation de signaux Doppler tolérants en trajectographie active; essais en mer. Journal de Physique IV Proceedings, 1994, 04 (C5), pp.C5-1137-C5-1140. 10.1051/jp4:19945251. jpa-00252940

\section{HAL Id: jpa-00252940 https://hal.science/jpa-00252940}

Submitted on 1 Jan 1994

HAL is a multi-disciplinary open access archive for the deposit and dissemination of scientific research documents, whether they are published or not. The documents may come from teaching and research institutions in France or abroad, or from public or private research centers.
L'archive ouverte pluridisciplinaire HAL, est destinée au dépôt et à la diffusion de documents scientifiques de niveau recherche, publiés ou non, émanant des établissements d'enseignement et de recherche français ou étrangers, des laboratoires publics ou privés. 


\title{
Utilisation de signaux Doppler tolérants en trajectographie active ; essais en mer
}

\author{
M.E. ZAKHARIA et F. JOLY*
}

CPE-ICPI Lyon, LASSSO Laboratoire d'Acoustique, Systèmes, Signaux et SOnar, 25 rue du Plat, 69288 Lyon cedex 02, France

* CPE-ICPI Lyon, LTS Laboratoire de Traitement du Signal, 25 rue du Plat, 69288 Lyon cedex 02, France

\begin{abstract}
The precision and the range of currently used trajectography system is mainly noiselimited. Increasing system performance has to go through the use of matched filtering. As, aside ambient noise and multipath, the Doppler effect is the most important disturbance encountered in the case of trajectography, the signals used have thus to be Doppler tolerant ones and any processing scheme has to take into account this phenomena at every step. In this paper, we describe a new system using a special type of linear period modulated chirps well known for their performance in the case of constant speed. As there is no optimal solution, in the case of constant acceleration, the same signals, although sub-optimal, have been used and the performance loss remains acceptable. The paper describes sea experiment using this type of signals associated to real-time digital filters. The results obtained with the improved acoustical system are compared to the data issued from surface positioning systems (radar, optics, DGPS) associated to a short baseline and a ship positioning. The results of both trajectography are coherent within a range of few metres.
\end{abstract}

\section{INTRODUCTION.}

L'augmentation de la précision de positionnement en trajectographie active passe par la réduction du bruit et, par conséquent, par l'utilisation de filtres adaptés aux codes émis par les mobiles. En plus du bruit et des trajets multiples, l'effet Doppler dû au mouvement des mobiles est un phénomène perturbateur important qui peut induire, en plus d'un biais, une chute du rapport signal sur bruit.

La vitesse élevée des mobiles impose la nécessité d'optimiser les codes utilisés en vue de minimiser les pertes de performance. Dans le cas de vitesse constante, plusieurs travaux ont montré l'intérêt des signaux à modulation linéaire de période[1], [2]. Dans le cas de vitesse variable (accélération constante) ces même signaux (quoique sous-optimaux) possèdent toujours des propriétés intéressantes [1], [3]. On se propose, dans ce travail de mettre en oeuvre de tels signaux dans des conditions réelles et de décrire les résultats d'essais en mer effectués sur les polygones de trajectographie TRÉMAIL. Deux mobiles ont été trajectographiés, l'un sur une perche et l'autre sur un poisson remorqué. Afin de chiffrer la précision des mesures acoustiques, un système complexe de positionnement a été utilisé: base courte, capteur de pression, centrale de cap-roulis-tangage, GPS différentiel, répondeur radar et positionnement optique.

Nous décrirons les essais entrepris ainsi que le système de traitement et de post-traitement utilisé et qui a permis une datation précise des réponses des mobiles (quelques mètres de précision à quelques kilomètres de distance). Les résultats d'essais seront enfin présentés et analysés. 


\section{SIGNAUX UTILISÉS.}

Des travaux antérieurs [1], [2] ont montré l'intérêt des signaux à modulation linéaire de période (modulation hyperbolique de fréquence) en présence de Doppler. Ces signaux, optimaux en présence d'une vitesse constante (pas de biais, minimisation de la perte de contraste en sortie de filtre adapté) restent intéressants dans le cas d'une accélération constante [3] (biais et perte négligeables). De tels signaux sont définis par leur expression fréquentielle:

$$
\left.\left.S(v)=A \cdot \exp \left[-\ln 2\left(v / v_{0}\right) / \operatorname{lng}\right] \cdot \cos 2 \pi b \ln v / v_{0}\right) / \ln g\right]
$$

où $v 0, \mathrm{~g}$ et $\mathrm{b}$ sont des paramètres intrinsèques au signal définissant respectivement la fréquence centrale, la bande relative et la durée du signal.

\section{ESSAIS EN MER}

\subsection{Description des essais.}

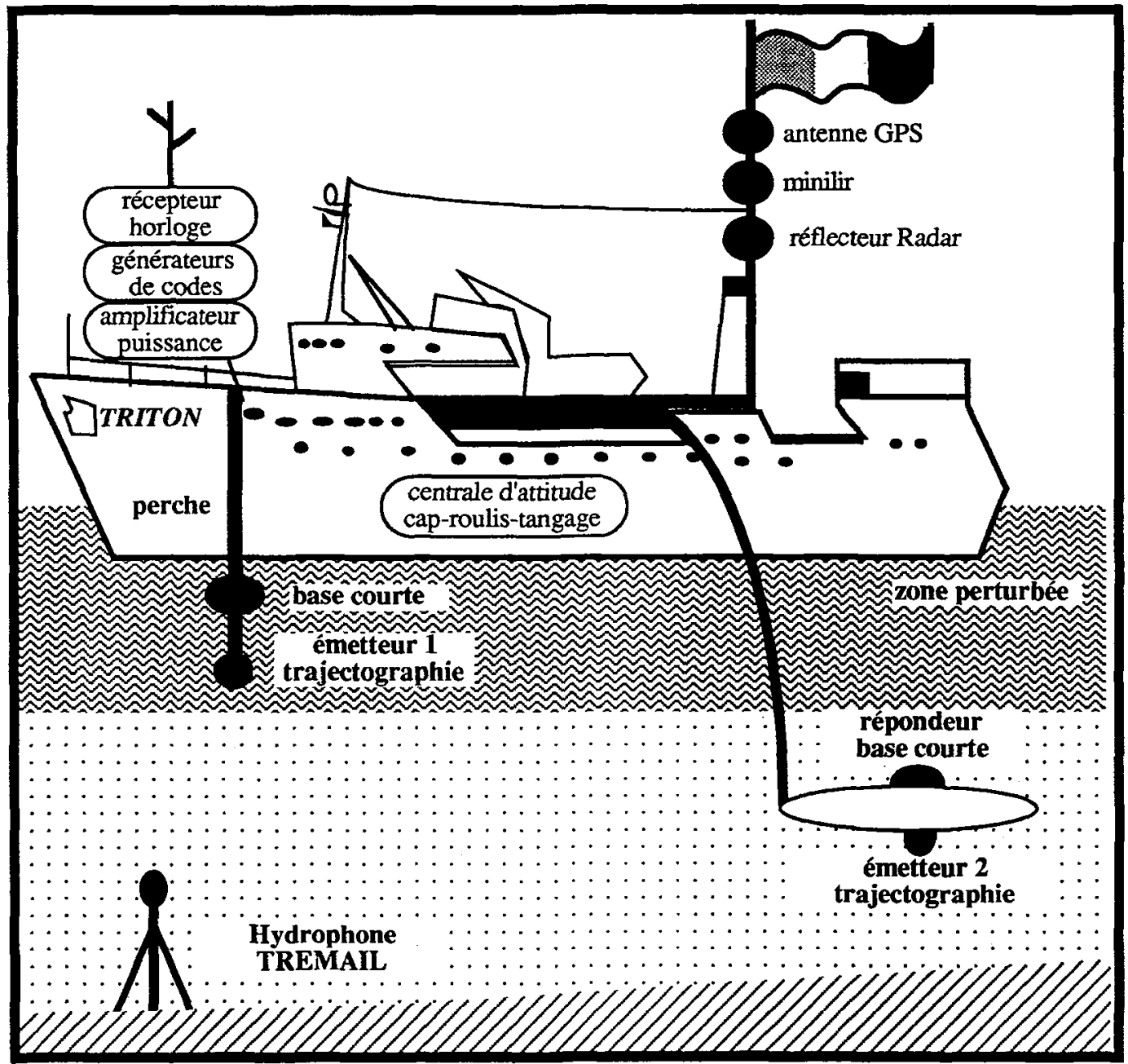

Figure 1: Description des essais 
Les signaux décrits, ainsi que les filtres adaptés associés (en temps réel), ont été utilisés lórs d'essais en mer. Deux mobiles ont été trajectographiés: le premier mobile était fixé à une perche et donc. solidaire du navire, le deuxième était situé sur un poisson remorqué (afin d'éviter la zone perturbée en surface). Afin de pouvoir valider les données de trajectographie acoustique, il a été nécessaire de mettre au point un système précis de positionnement de surface et de positionner les mobiles par rapport à la surface afin d'obtenir une trajectoire de référence. Pour cela, il a fallu disposer sur le navire de surface de plusieurs équipements:

- Système GPS différentiel, réflecteur radar et source infrarouge permettant de positionner

plusieurs points situés sur le mat du navire. Grâce aux moyens installés à terre, on a pu ainsi obtenir trois types de trajectoire de surface: GPS, radar et optique (théodolite de poursuite).

- Base ultracourte afin de positionner le poisson remorqué par rapport à la perche.

- Un système de Cap-Roulis-Tangage situé au centre de gravité du navire et permettant de

fusionner les données en provenance de la surface avec les donnés de fond.

Chaque fois que les conditions de propagation radio le permettaient, le système d'horloge temps réel du bord était synchrone de celui de la trajectographie.

Au moyen de plusieurs changements de repère, l'ensemble décrit permet de positionner chaque mobile par rapport à un référentiel absolu (DGPS ou positionnement optique).

L'ensemble des essais est décrit par la figure 1.

\subsection{Filtrage adapté temps réel}

Plusieurs filtres numériques en temps réel ont été développés pour ces essais. Ils utilisent une architecture basée autour d'un microprocesseur spécialisé DSP 56001 associé à plusieurs contrôleurs de filtre numérique Motorola DSP 56200. Les performances de calcul en temps réel sont les suivantes:

- fréquence maximale d'échantillonnage: Féch $=47 \mathrm{kHz}$

- durée maximale de la réponse impulsionnelle: $34,15 \mathrm{~ms}$ ( 1600 points pour Féch $=47 \mathrm{kHz}$ ).

Ces filtres ont été positionnés entre l'arrivée des hydrophones et l'entrée du système actuel de trajectographie. La détection et le post-traitement sont effectués au moyen d'un montage hybride basé autour d'un micro-contrôleur permettant de disposer, en sortie, de signaux totalement compatibles avec le système TREMAIL existant : impulsion brève = signal, impulsion longue $=$ lever de doute .

\section{RÉSULTATS.}

Les datations des impulsions acoustiques ont été traitées au CEM en utilisant le logiciel de trajectographie existant. Par ailleurs, une trajectographie utilisant les moyens de positionnement en surface a aussi été réalisée. Pour plusieurs cas, l'erreur entre les deux trajectoires a pu alors être estimée. La figure 2 montre un exemple des écarts entre la trajectographie acoustique et celle du positionnement de surface (dz: écart en immersion, dh: écart horizontal). Il est important de noter que ces résultats proviennent de la comparaison de deux trajectoires absolues. Les erreurs observées sont la résultante des erreurs sur tous les dispositifs de positionnement, de trajectographie et de changement de repère. Pour la plupart des essais l'erreur résiduelle se manifeste sous forme de biais (de quelques mètres) et d'une variance inférieure à quelques mètres. Il est important de noter que les "pics" d'erreurs ne correspondent pas à des changements de trajectographie; cette observation porte à croire que ces pics proviennent płutôt d'erreurs cumulées sur les systèmes de positionnement de référence (et plus particulièrement la bàse ultracourte) plutôt que du système de trajectographie acoustique.

\section{CONCLUSION.}

Les essais réalisés ont montré, en mer et dans des conditions opérationnelles, la faisabilité d'un système de trajectographie utilisant la compression d'impulsion même dans le cas de trajectoire quelconque.

Ils ont permis de mettre en évidence le "bon comportement" des signaux choisis en présence simultanée de bruit, de trajets multiples et d'effet Doppler. Ils ont montré la possibilité d'émettre de tels signaux au moyen de transducteurs standard et de les traiter en temps réel.

Compte tenu des échelles de précision absolue (quelques mètres), il est difficile de savoir si les erreurs observées sont dues à des erreurs de datation, à la trajectographie acoustique ou au système de positionnement de surface. Les résultats sont cependant globalement satisfaisants car on constate que, malgré la complexité des essais et du dépouillement et la difficulté de fusion des données, la précision absolue de quelques mètres a pu être obtenue dans la plupart des cas. 


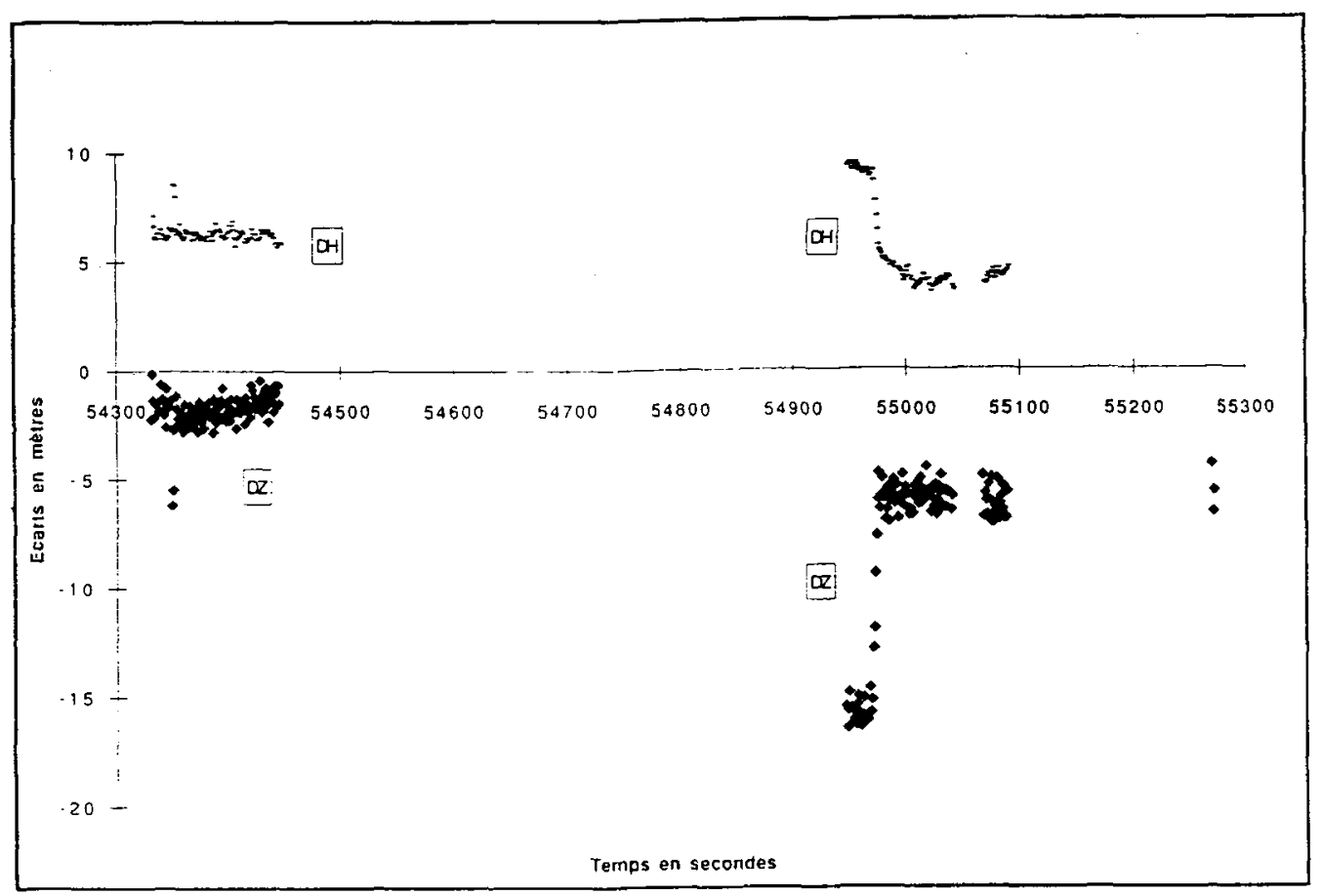

Figure 2: exemple de résultats (rajectographie acoustique du poisson remorqué)

\section{REMERCIEMENTS.}

Cette étude a été soutenue par le CTME (DGA/DRET/CTME). Le positionnement DGPS a été effectué par le LRBA (DGA/DME/LRBA). Le positionnement base courte a été effectué par le LDG (CEA/LDG). Les essais ainsi que les dépouillements des données de trajectographie ont été effectués en collaboration avec le Centre d'Essais de la Méditerranée, CEM (DGADME/CEM). Les essais en mer ont été réalisés à bord du TRTTON (GISMER).

\section{BIBLIOGRAPHIE.}

[1] : M. MAMODE: "Estimation optimale de la date d'arrivée d'un écho sonar perturbée par l'effet Doppler. Synthèse de signaux large bande tolérants." Thèse de Docteur Ingénieur (1981). Institut Polytechnique de Grenoble.

[2] R.A. ALTES: "Sonar for a generalized target description and its similarity to animal echolocation systems". J.Acoust.Soc.Am. 59, 97-105 1976.

[3] M.E. ZAKHARIA: "Étude et description de signaux tolérant à l'effet Doppler ". 13e Colloque GRETSI, pp. 597-600, septembre 1991. 